\title{
Las características determinantes de la administración de la justicia mercantil oral bajo el nuevo modelo de gestión judicial latinoamericano ${ }^{1}$
}

The determinant characteristics of the administration of the oral commercial justice under the new model of Latin-American judicial management

Roberto, Treviño Ramos²; Gustavo Daniel, Ortiz Téllez ${ }^{3}$; Héctor Hugo, Maldonado Salinas ${ }^{4}$

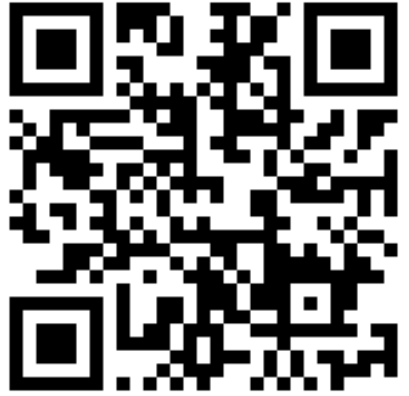

Fecha de recibido: $11-11-2020$

Fecha de aceptado: 29-02-2021

\section{(c) $)$ BY-NC-ND}

Esta obra está bajo una licencia de Creative Commons Reconocimiento-NoComercialSinObraDerivada 4.0 Internacional

\begin{abstract}
RESUMEN
Los países latinoamericanos poco a poco han comenzado a dejar atrás el sistema tradicional de justicia mercantil escrito, adoptando ahora el modelo oral similar a los aplicados en los países angloamericanos, que tienen como referente el derecho anglosajón con el fin de incrementar la seguridad y certidumbre a sus ciudadanos y usuarios entre los que se encuentran inversionistas extranjeros. El presente artículo es producto de una revisión documental cuyo objetivo consistió en establecer las características del nuevo modelo de gestión judicial en Latinoamérica. Se aplicó el método deductivo utilizando la técnica de investigación de revisión documental, el tipo de investigación es cualitativa, Como resultado de la investigación se consolida un marco literario-teórico científico, estableciendo ocho variables determinantes, las cuales fueron: eficacia, capacitación, tecnologías de información y comunicación, transparencia, especialización, unificación de criterios y la expedites.
\end{abstract}

Palabras claves: Administración Pública, Comercio, Gestión Judicial, Inversión extranjera, Latinoamérica.

\begin{abstract}
Latin American countries have gradually begun to leave behind the traditional written commercial justice system, adopting an oral model similar to those used in AngloAmerican countries, which have the common law as a reference in order to increase security and certainty to its citizens and users, including foreign investors. The following paper is a product of a documentary review, whose objective seeks to establish the characteristics of the new model of judicial management in Latin America. The deductive method was applied using the documentary review research technique, the type of research is qualitative, as a result of the research, a scientific literary-theoretical framework is consolidated, establishing eight determining variables which were: efficacy, training, information and communication technologies, transparency, specialization, unification of criteria and expedites.
\end{abstract}

Keywords: Commerce, Foreign investment, Judicial Management, Latin America, Public Administration

Cómo referenciar este artículo:

Treviño., R., R., Ortiz., T., G., \& Maldonado., S., H. (2021). Las características determinantes de la administración de la justicia mercantil oral bajo el nuevo modelo de gestión judicial latinoamericano. Revista Politica Globalidad y Ciudadanía, 7(14), 181-205. https://doi.org/10.29105/pgc7.14-9

\footnotetext{
${ }^{1}$ Este artículo es producto del proyecto "Las características determinantes de la administración de la justicia mercantil oral bajo el nuevo modelo de gestión judicial para los negocios y la inversión en Nuevo León” Iniciado en 2016 y finalizado en 2020.

${ }^{2}$ Universidad Autónoma de Nuevo León, Monterrey, México. Doctor en filosofía con orientación en Relaciones Internacionales, Negocios y Diplomacia por la Universidad Autónoma de Nuevo León. Profesor titular de la Facultad de Políticas y Relaciones Internacionales en la Universidad Autónoma de Nuevo León. Correo: roberto_tr2003@yahoo.com.mx. ORCID: https://orcid.org/0000-0001-8763-8557.

${ }^{3}$ Universidad Autónoma de Nuevo León, Monterrey, México. México. Maestro en Relaciones Internacionales por la Universidad Autónoma de Nuevo León. Correo: gustavo.ortiz.tellez@gmail.com. ORCID: https://orcid.org/0000-0002-4340-2232.

${ }^{4}$ Universidad Autónoma de Nuevo León, Monterrey, México. Maestro en Ciencias Políticas por la Universidad Autónoma de Nuevo León. Doctorante en filosofía con orientación en Relaciones Internacionales, Negocios y Diplomacia en la Universidad Autónoma de Nuevo León. Correo: hector.mldo@gmail.com. ORCID: https://orcid.org/0000-0003-0386-4253.
} 


\section{1.- INTRODUCCIÓN}

El presente estudio comprende un análisis de las características del nuevo método de administración de los juzgados orales mercantiles latinoamericanos denominado "Gestión Judicial", el cual se enfoca en identificar los principales factores que permiten una mejora en los servicios y características que orbitan el desempeño de los casos judiciales.

Un factor de suma importancia que es considerado en la inversión económica a nivel micro y macro es la eficacia en el desarrollo de los procesos judiciales del lugar donde se pretenda desarrollar el proyecto de inversión. Independientemente que los países y/o estados realicen políticas orientadas a atraer inversionistas a la entidad, en la mayoría de los casos estas políticas se ven opacadas en la medida de la eficiencia que su sistema judicial posee, alejando con ello a posibles inversores en la entidad. Así pues, el motivo de este análisis fue determinar las características esenciales que debe poseer este el nuevo modelo de trabajo para la justicia mercantil, para que este provea beneficios o ventajas que favorezcan o contribuyan a aumentar la realización de operaciones comerciales sometidos a la competencia de los juzgados orales mercantiles latinoamericanos, y mejorar la percepción del inversionista o de quien busque realizar negocios en Latinoamérica.

Además de los factores generalmente considerados por los inversionistas son: el tamaño de mercado, los riesgos (políticos y económicos), la apertura comercial (a las importaciones y exportaciones), la estabilidad económica (volatilidad del tipo de cambio, inflación), las políticas comerciales (nivel de protecciones arancelarias, tasa de impuestos), y otras ventajas comparativas (disponibilidad de recursos naturales, mano de obra calificada, etc.)

Según el Banco Mundial (2020) otro factor de suma importancia al desarrollar una inversión es la eficacia en el desarrollo de los procesos judiciales del lugar donde se pretenda realizar. Independientemente que los países y/o estados realicen políticas orientadas a atraer inversionistas, en la mayoría de los casos estas se ven limitadas en la medida de la eficiencia que su sistema judicial posee, alejando o atrayendo con ello a posibles inversores. Así pues, el motivo de este análisis es determinar las características esenciales de que debe esta investido el nuevo modelo de trabajo para la justicia mercantil

oral, para que este provea los beneficios o ventajas que favorezcan o contribuyan a aumentar la realización de operaciones comerciales sometidos a la competencia de los juzgados mercantiles orales 
latinoamericanos, y mejorar la percepción del inversionista o de quien busque realizar negocios en Latinoamérica.

Actualmente, en América latina no existen herramientas que midan la eficiencia operativa de las nuevas reglas de oralidad aplicadas a los asuntos de carácter comercial, esta investigación encuentra relevancia en ello, además la necesidad de este proyecto se justifica en razón de aglutinar evidencia teórica de los resultados prácticos de estos estructuras operativos, y describe a los posibles inversionistas las bondades de la solución de conflictos comerciales desahogados mediante modelos que operan bajo "gestión judicial" de aquellas inversiones que desafortunadamente caigan en incumplimiento de obligaciones, y justificar un elemento más para determinar la zona más ideal para realizar un inversión.

La pregunta de investigación es: ¿Cuáles son las características relevantes de los modelos de gestión judicial que permiten mejorar la justicia mercantil para motivar las inversiones en Latinoamérica? Y como objetivo general: Identificar las características del nuevo modelo de gestión judicial en Latinoamérica.

\section{2.-FUNDAMENTO TEÓRICO}

El desarrollo de la investigación inició con una búsqueda de documentos de investigación relacionados con el tema en bases de datos. Debido a que este modelo de Gestión Judicial es reciente, la literatura científica es escasa aún. Como resultado se encontró como antecedentes los trabajos de Linares (2008), Riego (2003) y Opazo (2014). Dichos trabajos si bien investigaban la gestión judicial, sus resultados estaban enfocados en diversos aspectos particulares.

El trabajo de Linares (2008) estudió el panorama en el cual se ha desenvuelto América Latina en cuanto a las políticas públicas existentes y que poco a poco se han ido reformando para mejorar la eficacia del sistema de gestión judicial en general. Sin mencionar algún entorno o juzgado en específico, nos habla sobre la necesidad de todos los países de América Latina de reformar sus políticas, ya que el problema reside en que según su conclusión no existe un adecuado control ciudadano y parlamentario sobre la administración de las políticas públicas de justicia, en donde la falta de rendición de cuentas del Poder Judicial sobre el Legislativo trae como resultado una acumulación de poder en una sola persona,

que en este caso sería el juez. Para Linares, la cuestión de la eficiencia y la rendición democrática de cuentas sigue siendo una cuenta pendiente en la gestión judicial. 
Treviño., R., R., Ortiz., T., G., \& Maldonado., S., H.

El trabajo de Riego (2003) fue un estudio comparativo entre los distintos juzgados en materia procesal penal entre los diversos países de América Latina antes y después de sus reformas en oralidad penal y el surgimiento de la gestión judicial de esa materia. En el estudio menciona sobre cómo la eficacia en los tribunales en materia procesal penal ha tenido frutos después de que al juez migrara del llamado principio inquisitivo, (en el cual el juez es el que lleva gran parte de la carga administrativa, así como la de investigación del caso al diverso modelo acusatorio. Lo anterior, según su dicho, traía como resultado una enorme carga de trabajo para el juez del modelo inquisitivo y propiciaba que se presentaran casos de anomalías debido a que los secretarios del juez, al no ser seleccionados por un organismo autónomo, sino por este mismo, permitían que se dieran con más frecuencia posibles actos de mala fe.

Por último, el trabajo de Opazo (2014) fue una investigación que buscó evaluar el sistema judicial chileno en cuanto a la implementación de herramientas que le permita desenvolver sus funciones con eficiencia, esto debido a que en Chile se han implementado en los últimos años reformas para una mejoría en la gestión judicial en el país. En esta investigación el autor se dedica a estudiar las variables que han permitido un mejor funcionamiento en el Poder Judicial chileno, así como su mejoría con el paso de los años, además de utilizar literatura científica de otros países relacionados con el tema para respaldar todos los puntos que se tocan en su estudio.

\section{Características determinantes del nuevo modelo de gestión judicial latinoamericano}

La revisión de literatura que aborda las características determinantes del nuevo modelo de gestión judicial, dando como resultado siete características más destacables, y arrojaron resultados positivos siendo estas las variables asociadas a la eficacia, la capacitación, el uso de las tecnologías de la investigación (TIC), la transparencia, la especialización, la unificación de criterios y la expedites; variables que fueron las sobresalientes en artículos y tesis realizadas en Chile, El Perú, Colombia, Argentina, Washington D.C. y México, en donde se buscaron y analizaron las características bajo diferentes modelos, tanto en el procesal como el civil y el mercantil.

\section{Eficacia}

Los elementos que componen la variable de eficacia son: La experiencia de los jueces en la organización; la participación de la sociedad civil en los procesos de selección de los jueces; la transparencia en los procesos de selección de los jueces; cuántas causas terminan por cada causa que ingresa en los tribunales; la separación de poderes, el management (introducción de técnicas de dirección para las empresas); la 
apertura a los nuevos instrumentos traídos gracias a la globalización; y los avances lentos debido juristas reacios al cambio.

La variable de Eficacia ha sido estudiada por las investigaciones de Linares (2008), Opazo (2014), Castro y García (2006), Riego (2003), Salazar (2014), Consentino (2009), Gregorio (2006), Vargas (2003), García (2012), Biebesheimer y Payne (2001), Binder (2000), Buscaglia (1997) y Baytelman y Duce (2003).

Linares (2008) examinó la variable de la Eficacia realizando un estudio acerca del mal estado en el que se encontraban las instituciones en la República de Argentina. En el estudio analiza la forma en que todos los actos judiciales de cualquier materia (mercantil, civil o procesal) no podían seguir el debido proceso ni llegar a una resolución debido al mal manejo que tenían los jueces sobre la organización de sus tribunales. Esto, sumado a una incapacidad por parte del personal que ahí labora, daba como resultado una ineficacia sobre los procesos judiciales. Concluye que después de las reformas implementadas en 2001, las cuales introdujeron la participación de la sociedad civil en los procesos de selección de los jueces de dicho tribunal, alentaron la adopción de prácticas institucionales que mejoraron la transparencia del funcionamiento de la Corte Suprema y de todas las instituciones que dependían de la misma. Esto generó un precedente de lo que se terminó llamando "Gestión Judicial”, en la cual —en este caso- la sociedad civil, conformada por personas capacitadas, organizaba y dictaminaba la conformación de los jueces en los tribunales. Cabe destacar que estas reformas en un inicio modificaron la Corte Suprema, pero los resultados llevaron a transformar todo el sistema judicial con el paso de los años.

El estudio de Opazo (2014) habla sobre las reformas llevadas a cabo en la gestión judicial del sistema chileno, en el cual aporta y habla sobre la importancia que tiene la eficacia en todos los casos judiciales. Este trabajo, además aportó un indicador para medir el volumen de eficiencia que tienen los diferentes juzgados sin importar la materia en la que se enfoquen, es así que para el investigador, la forma de medir qué tan eficiente son los juzgados es medir la cantidad de causas que llegan a término en los tribunales que las tramitan (como los de Familia y Letras del Trabajo), la cantidad de providencias y audiencias en los tribunales que sólo satisfacen requerimientos de parte (Juzgados de Garantía y Cobranza Laboral y Previsional), y la cantidad de audiencias de juicio en los Tribunales de Juicio Oral en lo Penal. El mismo indicador, pero ponderado en relación con el ingreso, dará lugar a la tasa de término (cuántas causas terminan por cada causa que ingresa). Una tasa de término igual a 1 indica equilibrio de ingresos y términos y, en consecuencia, estabilidad en el stock de causas o requerimientos pendientes; 
Treviño., R., R., Ortiz., T., G., \& Maldonado., S., H.

una tasa mayor a 1 indica disminución del stock; y menor que 1, un aumento del stock, es decir, mide las entradas y las salidas.

El nivel de términos (casos concluidos) también se puede ponderar por dotación a efectos de medir la cantidad de términos por juez y/o por funcionario. Por último, los términos por juez se pueden confrontar con los ingresos por juez, y así obtener una tasa de término por juez. En el estudio de Castro y García (2006) utilizaron los indicadores anteriormente mencionados para poder medir los resultados de la reforma procesal civil en Chile, llegando a la conclusión de que aún faltaba mucho por hacer en cuanto a este ámbito, esto debido a que los resultados fueron desfavorables aún y con la reforma implementada en el país.

El estudio de Salazar (2014) se enfocó en las reformas planteadas en el sistema judicial del Perú, en el cual llega a la conclusión de que la justicia peruana comenzó a tener eficiencia a partir de las reformas que trajeron como resultado la separación definitiva del Poder Judicial de los demás poderes del Estado peruano. El autor argumenta que sólo de esta manera cualquier país podría conseguir poseer eficacia y llegar a la justicia mediante el poder independiente, y que es de esta manera como la gestión judicial en el Perú ha podido sobresalir por encima de los países que no poseen este modo de operar. Esto también coincide con el estudio de García (2012), en la cual examinó las reformas llevadas a cabo en los juzgados chilenos para la materia familia, y una vez que se llevaron a cabo las reformas relacionadas con aspectos administrativos, aumentaron la eficacia en la gestión judicial.

El estudio de Consentino (2009) consistió principalmente en proporcionar las herramientas con las cuales se podría llevar a cabo las reformas para conseguir una mejor gestión judicial en los sistemas de impartición de justicia que aún seguían con el modelo considerado por algunos autores como obsoleto. Entre las sugerencias que el autor propone usar están: el uso las tecnologías de la administración y el management de las organizaciones, y de la información y las comunicaciones; propone que a través del uso de estas herramientas, los países conseguirán una mejor gestión judicial.

El estudio de Gregorio (2006), por su parte, realizó un análisis general de las reformas judiciales que han ocurrido en América Latina, dando a conocer además los resultados que obtuvieron los países que las implementaron. En él llegó a la conclusión que todos los avances en la gestión judicial se lograron gracias al proceso de la globalización, el cual trajo consigo numerosos tipos de tecnologías que facilitaron a los jueces llevar a cabo la gestión judicial, y que gracias a la implementación de estas tecnologías de la 
información, el propio sistema se vio obligado a implementar nuevos instrumentos, lo cual consiguió una total reforma del sistema judicial en muchos países de América Latina; todos estos cambios lograron una mayor eficacia en los casos en cualquier tipo de materia.

El estudio de Vargas (2003) realizó un análisis de varios países de América Latina, que durante los años noventa implementaron reformas hacía la gestión judicial. En el estudio argumenta que estos cambios, a pesar de ser positivos, sí poseen una fuerte resistencia para algunas personas que denomina "de la vieja guardia", las cuales son personas reacias al cambio, y por ende, ralentizan los efectos y los beneficios de las reformas judiciales, por lo cual, la estrategia de cambio de estructura no logra ser lo suficientemente amplia ni comprensiva, lo cual anula sus efectos a gran escala. Pero a pesar de lo anterior, si encuentra otras consecuencias que él denomina como positivas, como el hecho de que hoy en día gracias a esa transparencia y eficacia en los procesos es posible tratar con los jueces y ministros de cortes sobre temas que antes era imposible tocarlos, de tal manera que apoyándose de las conclusiones negativas y positivas de los estudios de Baytelman y Duce (2003) que analizó estos cambios en Chile, y los estudios de Biebesheimer y Payne (2001), Binder (2000) y Buscaglia (1997), cuyos estudios realizados con la intención de analizar la situación de las reformas en América Latina.

\section{Capacitación}

Los elementos que componen la variable capacitación son: La integración de organizaciones civiles en el sistema judicial; la integración de expertos; la restructuración de la organización judicial con los modelos actuales de la administración; la enseñanza a los jóvenes juristas sobre los principios básicos de la administración; la sensibilización de las personas; la existencia de canales de comunicación en la organización; el sistema de educación; la formación y desarrollo de recursos humanos en la formación de los juristas; el entrenamiento en otras ciencias; y las técnicas útiles para el mejoramiento de la Administración de Justicias.

La variable de Capacitación ha sido estudiado principalmente en las investigaciones de: Salazar (2014), Consentino (2009), Palma (2017), Hernández (2017), Bielsa y Brenna (1996), Palma (2007), Duce (2004), Garavano y Luis (2002), Acuña y Gabriela (2001) y López (2005).

Como se había mencionado anteriormente, el estudio de Salazar (2014) llegó a la conclusión de que el éxito del sistema judicial peruano fue por las reformas implementadas. Esta separación de poderes trajo consigo que el sistema judicial peruano integrará organizaciones civiles y expertos en la materia 
Treviño., R., R., Ortiz., T., G., \& Maldonado., S., H.

que pudieran servir como consultores y capacitadores de todos los individuos que, de alguna manera u otra, eran partícipes de la gestión judicial, esto para que siempre contaran con los principios básicos de lo que era la gestión judicial y cómo lograr la mayor eficacia posible en todos los casos en los que estuvieran involucrados estos individuos.

En el estudio de Consentino (2009), como parte del cambio que la sociedad reclama y que espera de la gestión del poder judicial, este llega a la conclusión que se deben reformar tres piezas importantes dentro del aparato de gestión judicial; estos son: la organización, los integrantes y operadores de justicia, y la gestión, teniendo como factor en común que para que estas tres piezas funcionen perfectamente, es necesaria la capacitación de las personas que lo llevan a cabo para una eficacia en la gestión judicial.

El estudio de Palma (2017) argumenta que la capacitación es criticada por los juristas, ya que ellos no están acostumbrados a recibir esta orientación al momento de organizarse, razón por la cual, la solución para no lidiar con este problema es que, desde principios de siglo, las escuelas juristas han comenzado a introducir estas ideas en los jóvenes estudiantes para revertir ese déficit carente de capacitación en la gestión judicial para lograr ese cambio cultural en la disciplina del derecho, coincidiendo con el estudio de Acuña y Gabriela (2001), el cual se dedicó a analizar como la capacitación impactó en América Latina, dando resultados favorables en países como Argentina, Brasil y Chile.

El estudio Hernández (2017) argumenta que es necesario la sensibilización de las personas y las instituciones involucradas en los organismos jurisdiccionales, si no, no se podrá desarrollar de manera acertada el proceso de comunicación, información y capacitación en la población judicial. Esta podría ser una limitación en la eficiencia en la gestión del poder judicial.

En el estudio de Bielsa y Brenna (1996) se evalúa la manera en la que está organizado el poder judicial. En el estudio argumentan que existen muchos indicadores que demuestran el poco aprecio de los representantes electos por el servicio de Justicia; entre estos indicadores tenemos la insuficiencia de las retribuciones y el acceso restringido, cuando no nulo, a la tecnología. Entre una gama muy amplia de desatenciones, las autoras concluyen que es necesario la apertura en la carrera que incluya ejes como sistemas de educación, formación y desarrollo de recursos humanos; de capacitación, formación complementaria y perfeccionamiento de los conocimientos jurídicos; de especialización y dominio de las técnicas judiciales; y de aprendizaje y entrenamiento en otras ciencias y técnicas útiles para el mejoramiento de la administración de justicia. 
Para el estudio de Palma (2007), la capacitación es el componente insustituible de todo proceso de reforma judicial al que se aspire a dar eficiencia; resultados exitosos sólo podrán producirse a partir de su óptima utilización por actores del sistema adecuadamente capacitados.

El estudio de Duce (2004) analiza la antigua gestión de los tribunales en Chile y llega a la conclusión de que la estructura se caracterizaba por el hecho de que cada tribunal era una unidad de trabajo a cargo de un juez que contaba con el apoyo de un secretario abogado y de varios empleados administrativos de baja capacitación. Llegó a la conclusión de que una vez que se realizaron las reformas en la gestión del poder judicial, se logró un aumento en la eficacia gracias a que el personal comenzó a ser capacitado, algo en lo que antes carecía la mayoría de los administrativos.

El estudio de Garavano y Luis (2002) analizó las necesidades que Argentina necesitaba para mejorar su gestión judicial. Concluyeron por medio de encuestas que la escasa capacitación era una de las grandes deficiencias con las que contaban los tribunales, razón por la cual era necesario reformar el sistema en este rubro, así como seguir aplicando encuestas a los usuarios para valorar la eficiencia y seguir mejorando con el tiempo la gestión judicial.

El caso de México es muy particular como cuenta el estudio de López (2005). El problema de México fue que durante las décadas de los ochenta y noventa este no pudo reformar su sistema de recursos humanos en sus diferentes áreas debido a que otros problemas de índole económica y de corrupción se presentaron, razón por la cual México llegó tarde a las reformas de gestión en el poder judicial.

\section{Uso de las Tecnologías de información y comunicación}

Los elementos que componen la variable de las tecnologías de información y comunicación son: La implementación de las TIC en los trámites tanto para los inversionistas, como para los jueces y sus allegados; la complejidad del uso de las nuevas tecnologías; la resistencia al cambio de las personas formadas en la antigua gestión judicial; la formación tradicional de los abogados y de los jueces ajenos a las TIC; el nulo conocimiento de conceptos de informática, administración y toma de decisiones; las presiones y los aspectos comerciales que rodean la informatización; los aspectos comercialices electrónicos; el temor de compartir información electrónica de los juzgados a través de la red; la creación de las escuelas juristas virtuales para introducir las TIC en los jóvenes juristas en formación; y la importancia de la modernización los sistemas de los propios tribunales. 
Treviño., R., R., Ortiz., T., G., \& Maldonado., S., H.

La variable de uso de Tecnologías de Información y la Comunicación fue una variable destacada en los estudios de Salazar (2014), Consentino (2009), Gregorio (2006), Rodríguez (2013), Londoño (2010), Alonso (2009), Pineda (2007), Jiménez (2005), Bielsa y Brenna (1996), Dory (2009), Garavano y Palma (2002), Contini y Velicogna (2012) y Castells (1998).

Desde la década de los ochenta comenzó el furor de introducir la tecnología en las organizaciones empresariales. "El ambiente en que se desenvolvían los gerentes sufrió cambios drásticos: la actividad empresarial cobró un carácter más global, se modificó dramáticamente la composición de la fuerza de trabajo, las nuevas tecnologías promovieron sus propios cambios y funcionaron como un acelerador del ritmo general de las transformaciones" según se menciona en el estudio de Bielsa y Brenna (1996). El estudio de Castells (1998) expone que la globalización obligará, a la larga, a que todas las naciones en sus tres poderes (si se trata de democracia) adoptarán las TIC en sus procedimientos, ya que es parte del crecimiento económico de los países.

Continuando, el progreso de la tecnología con el tiempo se ha comenzado a integrar en todos los ámbitos del poder judicial. Según el estudio de Dory (2009), existe un eterno debate entre la no comprensión de los jueces y poderes judiciales en el uso de las TIC, mientras que el otro punto de vista es el hecho de que los tribunales procesan la información de diferente manera que en los demás ámbitos de la sociedad, por eso ha sido lenta la adaptación de las TIC’s en la administración judicial y en todo el poder judicial en sí.

El estudio de Salazar (2014) analizó el sistema judicial del Estado del Perú, tomando como conclusión que las reformas implantadas habían tenido resultados positivos para el país. Además, otro punto de los que destaca es que la era de la globalización trajo consigo la implementación de las tecnologías en diversos rubros, tanto en el sector privado como el público, de esta manera, las TIC's, también fueron utilizadas en la gestión judicial del Perú, y esto facilitó los trámites tanto para los inversionistas, como para los jueces y sus allegados.

El estudio de Consentino (2009) se enfocó principalmente en dar ciertas sugerencias para que los países restantes que aún no habían reformado su sistema judicial lo hicieran bajo estas reglas, y de esta manera, ofrece una serie de recomendaciones entre las cuales destaca el uso de las TIC's, que según su investigación, los países que las implementaron en sus modelos de gestión judicial facilitaron las operaciones para todos aquellos involucrados en esta temática.

190 Revista Política, Globalidad y Ciudadanía | Vol. 7, Núm. 14, junio - diciembre 2021 | ISSN 2395-8448 | http://revpoliticas.uanl.mx/ 
El estudio de Gregorio (2006) aborda los cambios que tienen los sistemas judiciales en América Latina en su gestión judicial, concluyendo en su estudio sobre algunos problemas a los que se han enfrentado los abogados y jueces debido a este cambio. Como puntos destacados tenemos: la complejidad de las nuevas tecnologías; su propia jerga; la resistencia al cambio; la formación tradicional de los abogados y de los jueces, que no incluye conocimientos de otras ciencias, en particular conceptos de información, administración, toma de decisiones, las presiones y los aspectos comerciales que rodean la informatización, algo en lo que también coincide el estudio de Rodríguez (2013), en el cual también argumenta que los juristas se resisten al cambio en cualquiera de sus ámbitos en los que estos laboren; esto supone la imposibilidad, en las condiciones actuales, de dar un marco jurídico apropiado al procedimiento electrónico que permita, por ejemplo, el desarrollo adecuado del expediente judicial, y de la desconfianza que esto provoca en las personas en el exterior como dentro de la administración al momento de utilizarlos.

Por último, el estudio de Jiménez (2005) sigue esta línea argumental, el cual expone que es un hecho que el retraso tecnológico ha sido una constante en la administración de justicia en las últimas décadas por toda América Latina, todo esto por la desconfianza que los juristas creen que puede ocasionar involucrar a las tecnologías de la información en su administración judicial.

Como parte de querer hacer evolucionar el ambiente en el cual se desenvuelven los juristas, ciertos países han emprendido acciones para acabar con la resistencia que comúnmente encuentran las tecnologías con los juristas, como por ejemplo, el estudio de Garavano y Palma (2002) se enfocó principalmente en analizar la escuela jurídica virtual en Argentina, la cual tiene por objetivo introducir desde el inicio de las carreras de los jóvenes juristas, las tecnologías de la información.

En el estudio de Londoño (2010) se reconoce esta resistencia de los juristas en la introducción de las TIC's en los ámbitos jurisdiccionales; sin embargo, no todo es negativo para el autor, ya que las TIC's también están sirviendo de puente para entablar una gran cantidad de relaciones jurídicas que ignoran las distancias y desafían los parámetros tradicionales, lo cual está dando orientación a las instituciones del ámbito jurídico a tener que adaptarlas en su organización.

En el estudio de Alonso (2009) se analiza cuáles son las demandas que exige la sociedad española ante la organización judicial, y uno de los puntos que destaca es la demanda de introducir las tecnologías de información como un instrumento facilitador que pueda agilizar los trámites y, de esta manera, 
Treviño., R., R., Ortiz., T., G., \& Maldonado., S., H.

resolver los problemas de la sociedad española, lo anterior puede influir en latinoamericana en relación a los lazos culturales e históricos existentes.

El estudio de Pineda (2007) habla sobre cómo las tecnologías de información han penetrado en la ciencia del derecho, en particular en la administración y procuración de la justicia, lo que ocasiona una profunda modernización en sus sistemas, tanto de control social, fiscalización, recaudación y en los propios tribunales. Los beneficios que trae esta introducción de las tecnologías es que la actividad gubernamental, y de impartición de justicia, sea más eficiente, más pronta y expedita.

El estudio de Contini y Velicogna (2012), por otro lado, hace un análisis de cómo las tecnologías de la información han estado implementándose en Europa en los últimos diez años, en los cuales ha tenido resultados positivos; sin embargo, concluye que el desarrollo de e-services en procedimientos judiciales tiene que enfrentar un nivel muy alto de complejidad en las áreas de regulación, organización, gobernanza y desarrollo. El entrelazamiento de tales factores lleva a un orden de complejidad que tiene muchos rasgos que son sustancialmente diferentes de ellos, de las otras innovaciones del sector judicial. Razón por la cual, el proceso de incorporación de las TIC en América Latina también debe de tener cierta paciencia para que puedan cumplirse en su totalidad las intenciones de la incorporación de las TIC.

\section{Transparencia}

Los elementos que componen la variable de transparencia son: La confianza en los individuos; la posibilidad de examinar el estado del caso; el seguimiento en los trámites; el sentimiento de cercanía con los jueces; el combate a la corrupción; la buena reputación; la atracción del país como posible foco de inversión; y el libre acceso a la información por parte de la sociedad civil, conocer el criterio jurídico que aplican los jueces para resolver los casos. El acercamiento de los estudiantes a la solución que los jueces dan a los temas trascendentes de la sociedad.

La variable de transparencia ha sido principalmente estudiada en las investigaciones de Binder (2008), Salazar (2014), Vallejo (2013), Franco (2017), Buergentha (2017), Castillo (2013), Toledo (2014), Orme (2017), Ackerman (2001), y Mejía (2001).

En su estudio, Binder (2008) habla sobre los cambios positivos que ha traído la transparencia al implementarla dentro de los cambios de la gestión judicial. La transparencia en su estudio provoca en la sociedad en general poder examinar el estado de un caso, darle seguimiento al mismo tiempo, y es así 
como los individuos poseen más confianza en todos sus casos judiciales, ya que hay más certidumbre sobre el desarrollo de los juicios.

El estudio de Salazar (2014), dedicado a examinar las consecuencias en los países que reformaron su gestión del sistema judicial, arroja como una de sus conclusiones que la transparencia trae consigo confianza en los individuos involucrados en los casos y, al igual que Vargas (2003), está de acuerdo con que esta transparencia provoca que las personas puedan hablar con los jueces y ministros de temas que antes no se atrevían debido a la falta de transparencia previamente inexistente en estas instituciones. Además, coincide con los estudios de Ackerman (2001) y Mejía (2001), en los cuales, ambos estudios exponían sobre las graves fallas que tenía el sistema judicial peruano debido a la falta de transparencia y por la corrupción existente en todas las áreas de la administración judicial.

El estudio de Vallejo (2013) habla sobre la carencia de transparencia en la gestión judicial mexicana, debido a que la mayoría de los estados de la República Mexicana siguen sin reformar las gestiones judiciales estatales; y, a pesar de que promueven la eficacia y la transparencia, esta no es posible de alcanzar debido a que no se tienen las herramientas adecuadas para ponerlas en práctica. Concluye que, si contamos con los instrumentos para ponerlas en práctica, será asimismo un mecanismo más para combatir la corrupción al interior del Poder Judicial de la Federación.

El estudio de Franco (2017) tiene como eje principal que la transparencia es un principio y un fin para la función jurisdiccional. Sin la transparencia nunca existirá una buena gestión judicial, razón por la cual para los Estados es sumamente necesario incluir la transparencia en las reformas judiciales que implementan para conseguir la buena reputación y que su país sea atractivo para invertir.

Por otro lado, el estudio de Buergentha (2017) realizó una comparación entre los diversos sistemas jurídicos y su forma de organización entre algunos de los países de América Latina, de esta manera, comparando países que han implementado el Nuevo Sistema de Gestión Judicial (Argentina, Chile y Perú y Panamá) con otros que aún no reforman en su totalidad su sistema de gestión judicial (Ecuador y México). El autor concluye que el nuevo Sistema de Gestión Judicial trajo consigo transparencia en los países, lo que provocó que estos fueran un país más atractivo para los inversionistas, lo cual favoreció a los países que optaron por reformar su sistema de gestión judicial.

El estudio de Castillo (2013) es un análisis más centrado en un sólo país; en este caso realizó un análisis de las reformas llevadas acabó en la República de Panamá, la cual trajo como resultado la 
Treviño., R., R., Ortiz., T., G., \& Maldonado., S., H.

independencia del Poder Judicial sobre los otros dos poderes; desde ese momento en adelante, la sociedad civil participó más, activamente, dentro de la gestión judicial, y lo que benefició al Poder Judicial fue el hecho de que se volvieron más transparentes con todos los casos que comenzaron a llevar a cabo bajo esta nueva gestión.

El estudio de Toledo (2014) es una investigación realizada en Paraguay, en el cual se emitió un informe acerca de los problemas de corrupción por los cuales pasa el país. El estudio argumenta que a pesar de tener instituciones que luchan en contra de la expansión de la corrupción en los sistemas del país, es necesario la independencia del Poder Judicial, así como la realización de una reforma para cambiar el sistema de gestión judicial de Uruguay, siguiendo de esta manera el ejemplo de los demás países de América Latina, la cual parece tener resultados positivos por el momento.

Desde una perspectiva más amplia, el estudio de Orme (2017) es un estudio que trata sobre la transparencia, en general, de los tres poderes. El autor da un repaso sobre la necesidad que tiene América Latina de reformarse en su totalidad para garantizar el libre acceso, y con ello, la transparencia en toda clase de instituciones.

\section{Especialización}

Los elementos que componen la variable de especialización son: La implementación de nuevos modelos basados en la separación de las actividades jurisdiccionales y administrativas; el entorno democrático garantizado; la abstinencia política de los jueces, el sistema jurídico modernizado; la calidad profesional; la disponibilidad social de conocimientos para la rápida dictaminación de la sentencia.

La especialización es una variable estudiada principalmente por Palma (2017), Vergara (2015), Ubertone (2006), Ledesma (2017), Torres (2014), Mora (2005), Vargas, Peña y Correa (2001) y Lisa (2014).

El estudio de Palma (2017) analiza desde un enfoque sistémico la modernización judicial en los países de la región latinoamericana, de esta manera es como el autor identifica dos corrientes: una basada en la capacitación en gestión para mejorar los modelos existentes, y la segunda es centrada en la implementación de nuevos modelos basados en la separación de las actividades jurisdiccionales y administrativas. El autor concluye que la segunda corriente es la recomendable para los países latinoamericanos ya que entre más independencia haya, se consigue que los individuos (administrativos) 
involucrados en la gestión judicial se especialicen; gracias a la especialización y gestión profesional de las tareas administrativas, se consigue una mayor eficacia, razón por la cual es recomendable que esta medida se lleve a cabo al reformarse el sistema de gestión judicial.

El estudio de Vergara (2015) habla sobre la necesidad de que todos los modelos de justicia en América Latina deben ser democráticos y, sobre todo, modernos. Esta modernización se conseguirá a base de la especialización y por la tendencia a dictar sentencias de acuerdo a principios; y democrático, tanto por la abstinencia política de sus jueces como por la observancia de las genuinas fuentes de derecho al dictar sus sentencias, ya que como dice el estudio de Lisa (2014), argumenta que cuando la estructura institucional y la ideología de un poder judicial se anclan en el ideal del apoliticismo, produce jueces capacitados y poco dispuestos a defender los derechos fundamentales y un estado de derecho, razón por la cual la democracia es fundamental para tener una eficiente organización judicial.

El estudio de Vargas, Peña, y Correa (2001) apoya la especialización, mencionando que gracias a usar este instrumento, a través de grupos pequeños, se ha conseguido un ahorro económico enorme, y sugiere su implementación en todas las áreas del poder judicial.

Por otro lado, el estudio de Ubertone (2006) habla sobre la importancia que tienen tanto los jueces como los administrativos que en la era de la globalización, los individuos encargados de este ámbito estén especializados en sus tareas ya que para el autor la sociedad demanda que es necesario esta especialización para la mejora constante de la calidad profesional de quienes desempeñan funciones judiciales.

En el estudio de Ledesma (2017) se aborda la problemática consistente en el que año con año son miles los casos que se deben de abordar por los jueces y sus secretarios, razón por la cual la gestión judicial se ve en la necesidad de responder con prontitud, creciente especialización y disponibilidad social de conocimientos sobre cómo llevar adelante organizaciones y su administración, y de esta manera, que los casos no se vean estancados por mucho tiempo.

Por otro lado, el estudio de Torres (2014) aborda el tema de las reformas judiciales llevadas a cabo en Brasil, específicamente sobre los jueces, ministros y sus secretarios para una eficiente gestión judicial sobre los procesos que han llevado a cabo, llegando a la conclusión respecto que la especialización de los secretarios en los juzgados ayudó a agilizar los casos que se iban presentando durante el año, dando como resultado una eficaz gestión judicial. 


\section{Unificación de criterios}

Los elementos que componen la variable de la unificación de criterios son: La agilidad en los procesos: la reducción de tiempos en los procesos; el aumento de la confianza en el sistema; la atenuación de la intervención de elementos extrajurídicos en las resoluciones judiciales; el ahorro económico tanto para los tribunales como para las personas que hace uso de los mismos; la disminución de barreras burocráticas; la prevención del daño antijurídico estatal; la eficaz defensa de los intereses estatales desde un ámbito técnico, organizacional y funcional de las instituciones encargadas de la defensa legal; la implementación de mecanismos alternos que solucionarán los conflictos entre la parte demandante y el Estado.

La unificación de criterios es una variable principalmente estudiada por Mora (2018), Delgado y Díaz (2011), Salazar (2014), Jiménez (2006), Rodríguez (2013), Estrada y Gleidy (2015), Manjónm (2008) y Alvarez (1993).

El estudio de Mora (2018) buscó proponer reformas para las juntas de los letrados de la administración de justicia. Entre las propuestas de las reformas que el autor sugiere, destaca la de desear la unificación de criterios en las juntas para una mejor agilidad en el ámbito procesal, y que esto se pueda expandir en los demás ámbitos del poder judicial, ya que esto reduce los tiempos de espera en los procesos que lleva día a día el poder judicial.

El estudio de Delgado y Díaz (2011) buscó señalar cuáles son los beneficios destacables de la unificación de criterios en el ámbito judicial, concluyendo que son más los beneficios que pérdidas el hecho de tratar de unificar las diferentes labores que él mismo realiza. Señala que entre las utilidades prácticas positivas se tiene el aumento en la confianza en el sistema y la atenuación de la intervención de elementos extrajurídicos en las resoluciones judiciales, además de destacar los méritos de carácter económicos que benefician tanto a los tribunales como a las personas que hacen uso de estos instrumentos.

El estudio de Salazar (2014) analiza la unificación de criterios del Estado peruano, en el cual ya fue implementada esta acción, sin embargo, en la investigación, recalca que los esfuerzos por unificar los criterios jurisdiccionales no han tenido los resultados deseados debido más que nada, por la burocracia que no permite que se realicen correctamente estas acciones, razón por la cual, a pesar de haber adoptado 
estos importantes acuerdos, no se pueden observar aún en la práctica judicial peruana, dejando en claro lo descuidado que está el poder judicial en Perú por la manera en que es abordada esa actividad.

El estudio de Jiménez (2006) es más práctico que los anteriores estudios, ya que en este se analiza la unificación de criterios que realizó la administración judicial española en el municipio de Pamplona. En dicho municipio, se optó por unificar las solicitudes de documentación clínica que se recibían en todo el territorio; anteriormente, eran seis los centros que recibían esa documentación, los cuales unos eran más eficientes que otros, pero algunos destacaban más en otros ámbitos, debido a que cada uno contaba con diferentes personas especializadas hasta cierto nivel, de tal manera que, una vez que se unificaron los criterios y sólo un centro se dedica a recibirlos, los resultados fueron más eficientes debido a que todas las personas estaban capacitadas y especializadas en cierta tarea, lo que suma un punto importante a lo positivo que es la unificación de criterios y lo que podría ser si se aplica esto a toda la gestión judicial.

En el estudio de Rodríguez (2013) se recalca que la unificación de criterios es algo que debe de realizarse en todas las administraciones judiciales de todos los países, esto debido a que la era de la digitalización permite una efectiva comunicación en la cual ya no es necesario que exista una diversificación de tareas, ya que la comunicación es más fácil y rápida, permitiendo que ciertas personas se ocupen sólo de ciertas tareas. Además, también recalca la necesaria unificación de algunos códigos como el civil y comercial, ya que con el paso del tiempo esto ha hecho que sean parecidos en algunos aspectos.

En el estudio de Estrada y Gleidy (2015), ambos autores analizaron cuáles fueron los factores que tuvo que implementar el Estado colombiano en medio de la crisis judicial por la que atravesó el país en la década de los 2000. El Estado colombiano creó la Agencia Nacional Jurídica del Estado, y esta se encargó, primero, de buscar las causas por las cuales se había originado la crisis; tales causas fueron, en primer lugar, la insuficiencia de recursos financieros, logísticos y de personal para implementar y fortalecer medidas de prevención del daño antijurídico estatal, la ineficaz defensa de los intereses estatales, desde un ámbito técnico, organizacional y funcional, de las instituciones encargadas de la defensa legal, la no implementación de mecanismos alternos que solucionaran los conflictos entre la parte demandante y el Estado; y la no unificación de criterios jurídicos para defensa Estatal. Una vez que se comenzó a tratar de mejorar todos los aspectos mencionados anteriormente, el Poder Judicial logró conseguir una base sólida para su actuación en todos los procesos que le correspondía actuar. 
Treviño., R., R., Ortiz., T., G., \& Maldonado., S., H.

Otro ejemplo es el del estudio de Álvarez (1993), en la cual se analizó la experiencia de los juzgados de Buenos Aires, en los cuales el autor argumenta dos puntos muy importantes, el primero era que el número de casos generados por accidentes de tránsito era ampliamente mayoritario; y, como segundo punto, observó que los montos indemnizatorios otorgados en estos casos diferían significativamente de un juzgado a otro, aun cuando los casos eran parecidos. La solución a esto fue crear una sola base de datos otorgada por la Cámara de Apelaciones, que permite recuperar casos con sentencia firme por medio de los datos de la víctima o de los reclamantes. Además, se descubrió que el sistema desarrollado resultaba muy útil para apoyar el proceso de mediación. Este es uno de los casos más antiguos que existen de la unificación de criterios; es un precedente de las reformas judiciales que se aplicaron en los siguientes años.

El estudio de Manjónm (2008) realizó una reflexión acerca de los diversos acuerdos y sugerencias en los cuales los magistrados celebran juntas en las que discuten y reflexionan sobre diversas sugerencias que podría plantear el Poder Judicial para obtener una mayor eficacia en todo su aparato judicial. Entre las diversas sugerencias que se hacen, la que más destaca es la de impulsar la unificación de criterios en todos los ámbitos del sistema judicial.

\section{Expedites}

Los elementos que componen la variable expedites son: Los mecanismos jurídicos a los que pueda acudir el individuo; la legitimidad democrática de los juzgadores, la reducción de cargas de trabajo; la modificación de la estructura orgánica; y las competencias de los tribunales.

La variable Expedites fue una variable que se destacó en las investigaciones de Peñaranda (2010), Nieto (2007), Amunátegui (2011), Ruiz (2010), Vivianco (2008), Cipoletta (2009), Raynaud (2009) y Pineda (2007).

Entendemos como expedito a algo "que carece de obstáculos, estorbos o inconvenientes" (William Collins Sons \& Co, 2005). El estudio de Peñaranda (2010) analizó los principios procesales del amparo constitucional. Lo que destaca en su estudio es que, para él, el amparo es un claro ejemplo de cómo obtener una justicia en forma expedita, lo que es fundamental para todo el poder judicial, ya que la justicia en forma es algo que se incluye en la Carta Magna de la mayoría de las naciones. 
El estudio de Nieto (2007) argumenta, como pilar fundamental, que la justicia y la confianza pública en ella son fundamentales para construir una sociedad más equitativa, es por eso que, a lo largo de su estudio, analiza diferentes artículos para establecer cuáles son los temas que deben ser reformados en México, en los cuales tuvo como conclusión que los que más destacan en los debates de los juristas para reformar la administración judicial son: la legitimidad democrática de los juzgadores; la expedites en la impartición de justicia; la reducción de cargas de trabajo; la modificación de la estructura orgánica; y las competencias de los tribunales; entre otros.

Como había mencionado anteriormente el estudio de Pineda (2007), la introducción de las tecnologías ha traído como beneficio, que, con el uso de esta, la actividad gubernamental y la de impartición de justicia puede ser expedita.

El estudio de Amunátegui (2011) fue dedicado a analizar la historia del poder judicial en Chile durante el periodo comprendido de 1965 a 1973, dicho período está dividido en etapas: la primera es comprendida antes del socialismo y, la segunda, es una vez ya se había establecido el gobierno socialista de Salvador Allende. El autor destaca que, durante la etapa previa a Allende, la justicia chilena estaba desorganizada; fue hasta una vez que Chile iba camino al socialismo cuando el nuevo partido político en el poder decidió reformar toda una organización judicial, siendo más expedita, es decir, sin obstáculos a la hora de que pusiera en acción sus atribuciones. Todo esto decayó con la dictadura de Pinochet, después de la década de los setenta. Sin embargo, en la actualidad, el Poder Judicial actúa como un mecanismo muy importante después de los turbulentos cambios que le tocó vivir en el siglo XX. El estudio de Vivianco (2008) continúa analizando el caso chileno, argumentando que la justicia chilena en la actualidad ha demostrado ser más expedita que en el pasado, ya que después de la dictadura se retomaron estos enfoques, y, además, se incorporó fácilmente a la era digital, logrando que Chile sea un ejemplo exitoso en la reforma de la administración judicial.

Por último, el estudio de Ruiz (2010) realizó un análisis de la administración de justicia enfocada en materia laboral y de seguridad social. El autor, en su estudio hace una crítica en el sentido que la investigación arrojó que México tiene enormes retrasos en reformar la administración de justicia, en especial enfocada en asuntos de carácter de seguridad social básica. Esto es un fenómeno común en toda América Latina, ya que los estudios de

Cipoletta (2009) en Argentina, y Raynaud (2009) en Nicaragua, llegaron a las mismas conclusiones en sus respectivos países, lo que da resultado una administración de justicia poco expedita. 


\section{3.-MÉTODO}

En base a lo explicado por Creswell (1994), el propósito de este estudio secuencial es la elaboración de un marco teórico literario que permita identificar las principales características para la correcta y eficiente gestión judicial bajo el nuevo modelo de justicia oral.

La investigación consistió en explorar teóricamente los diversos conceptos involucrados relacionados con la problemática, y subsiguientemente fue necesario respaldar las características determinantes necesarias para garantizar lo que es la gestión judicial ideal.

La presente investigación es cualitativa no experimental, exploratoria y documental, el modelo de investigación propuesto estriba en la investigación exploratoria y descriptiva de las variables determinantes de la administración de la justicia mercantil oral bajo el nuevo modelo de gestión judicial, así como el estudio de los resultados alcanzados a la luz de cada uno.

\section{4.-RESULTADOS}

En el contexto de la revisión de la literatura fueron identificados las siguientes características: eficacia, capacitación, uso de tecnologías de la información y comunicación, transparencia, especialización, unificación de criterios, y expedites (ver tabla 1) con el objetivo explorar las características determinantes en el nuevo modelo de gestión judicial oral en materia mercantil.

Como se puede observar en la tabla anterior, se presenta de manera visual las características que determinan el Nuevo Modelo de Gestión Judicial, así como las investigaciones en las que dichas variables han sido abordadas en los respectivos países latinoamericanos, creando un marco literario/científico del cual se pueda identificar de manera inmediata dichas características. 
Tabla 1.

Los factores determinantes de la nueva gestión judicial

\begin{tabular}{|c|c|c|}
\hline Característica & Autor & Lugar \\
\hline Eficacia & $\begin{array}{l}\text { Linares (2008) Opazo, (2014) Riego (2003) Salazar (2014) } \\
\text { Consentino (2009) Gregorio, (2006) Vargas (2003) Castro } \\
\text { y García (2006) García (2012) Biebesheimer y Payne } \\
\text { (2001) Binder (2000) Buscaglia (1997) Baytelman y Duce } \\
\text { (2003) }\end{array}$ & $\begin{array}{l}\text { Chile, Perú, Colombia, México, } \\
\text { Argentina, Washington D.C }\end{array}$ \\
\hline Capacitación & $\begin{array}{l}\text { Salazar (2014) Consentino (2009) Palma (2017) } \\
\text { Hernandez (2017), Bielsa y Brenna (1996) Palma (2007) } \\
\text { Duce (2004) Garavano y Luis (2002) Acuña y Gabriela } \\
\text { (2001) López (2005) }\end{array}$ & $\begin{array}{l}\text { Chile, Perú, Colombia, México, } \\
\text { Washington D.C, Argentina, Brasil }\end{array}$ \\
\hline Uso de TIC & $\begin{array}{l}\text { Consentino (2009) Gregorio (2006) Rodríguez (2013) } \\
\text { Londoño (2010) Alonso (2009) Pineda (2007) Jiménez } \\
\text { (2005) Bielsa y Brenna (1996) Dory (2009) Contini y } \\
\text { Velicogna (2012) Dory (2009) Garavano y Luis (2002) } \\
\text { Castells (1998) }\end{array}$ & $\begin{array}{l}\text { Argentina, Colombia, } \\
\text { México, Europa. }\end{array}$ \\
\hline Transparencia & $\begin{array}{l}\text { Binder (2008) Salazar (2014) Vallejo (2013) Franco } \\
\text { (2017) Buergentha (2017) Castillo (2013) Toledo (2014) } \\
\text { Azoulay (2017) Ackerman, (2001) y Mejía (2001) }\end{array}$ & $\begin{array}{l}\text { Chile, Perú, Colombia, México, } \\
\text { Panamá, Uruguay }\end{array}$ \\
\hline Especialización & $\begin{array}{l}\text { Palma (2017) Vergara (2015) Ubertone (2006) Ledesma } \\
\text { (2017) Torres (2014) Mora (2005) Vargas, Peña, y Correa } \\
\text { (2001) Lisa (2014) }\end{array}$ & $\begin{array}{l}\text { Chile, Perú, Colombia, México, } \\
\text { Argentina, Paraguay, España, Brasil }\end{array}$ \\
\hline $\begin{array}{l}\text { Unificación } \\
\text { criterios }\end{array}$ & $\begin{array}{l}\text { Mora (2018) Delgado y Díaz (2011) Salazar (2014) } \\
\text { Jiménez, (2006) Rodriguez, (2013) Estrada y Gleidy } \\
\text { (2015) Manjónm (2008) Álvarez (1993) }\end{array}$ & $\begin{array}{l}\text { España, Chile, Perú, Argentina, } \\
\text { Colombia }\end{array}$ \\
\hline Expedites & $\begin{array}{l}\text { Peñaranda (2010), Nieto, (2007) Amunátegui (2011), Ruiz } \\
\text { (2010) Vivianco (2008) Cipoletta (2009) Raynaud } \\
\text { (2009) Pineda (2007) }\end{array}$ & $\begin{array}{l}\text { España, México, Chile, Argentina, } \\
\text { Nicaragua }\end{array}$ \\
\hline
\end{tabular}

Fuente: Elaboración propia (2021).

\section{5.-CONCLUSIÓN}

El propósito de esta investigación consistió en identificar y analizar las características que determinan el nuevo modelo de gestión judicial en la administración de justicia mercantil para los negocios y la inversión en Latinoamérica. Primeramente, se realizó un análisis de los principales conceptos y el funcionamiento de todo lo que conlleva este ámbito en particular.

\section{De la comprobación cualitativa}


Treviño., R., R., Ortiz., T., G., \& Maldonado., S., H.

Una vez contextualizada la situación, se dio paso al desarrollo de la investigación en la identificación de las principales características a través de la literatura científica. Se consolidó un marco conceptual de distintas variables en las cuales dentro del mismo se agruparon los diferentes estudios que identificaron características en común. Todo esto presentado en el capítulo "Características determinantes del nuevo modelo de gestión judicial".

\section{De las limitaciones del estudio}

Para concluir esta investigación, es necesario puntualizar las limitaciones que se presentaron durante este estudio. La primera limitante fue la falta de información existente sobre este tema, razón por la cual se tuvo que investigar esta problemática en otros países tomando como referencia datos bibliográficos europeos.

\section{De las recomendaciones}

Esta investigación exhorta a interesados en el análisis de las características, ventajas y bondades de este tipo de modelos de impartición de justicia para que aporten nuevos estudios con datos cuantitativos de los diversos participantes para respaldar la fiabilidad de las características recolectadas en la literatura científica.

\section{REFERENCIAS}

Ackerman, R. (2001). Corrupción en el Poder Judicial Peruano: marco conceptual. Bioetica .

Acuña, C., \& Gabriela, A. (2001). La reforma judicial en América Latina: un estudio sobre las reformas judiciales en Argentina, Brasil,Chile y México .CLAD.

Alonso, J. (2009). Las Tic en la Justicia del Futuro. Ariel.

Alvarez, G. (1993). El enfoque empírico: un sistema de ayuda a la decisión judicial. En R. Guibourg (Ed.) Informática Jurídica Decisoria (pp. 191-210). Editorial Astrea.

Amunátegui, A. (2011). El protagonismo político del Poder Judicial entre los años 1965 y 1973. Revista de Derecho, 619 - 663.

Banco Mundial. (2020). Informe Doing Business. https://espanol.doingbusiness.org/es/data/exploretopics/enforcing-contracts/mexico

Baytelman, A., \& Duce, M. (2003). Evaluación de la Reforma Procesal. Centro de estudios de Justicia de las Americas y Facultad de Derecho de la Universidad Diego Portales.

Biebesheimer, C., \& Payne, M. (2001). Experience in Justice Reform: Lessons learned and elements for policy formulation. IDB.

Bielsa, R., \& Brenna, R. (1996). Recursos humanos, nuevas tecnologías y Poder Judicial . Informática y Derecho, (2), 709-766.

Binder, A. (2000). De las Repúblicas Aéreas al Estado de Derecho Ideas Para un Debate sobre la Marcha de la Reforma Judicial en América Latina. AD-HOC. 
Binder, A. (2008). ¿Cómo y sobre qué debe rendir cuentas el sistema judicial?. https://www.telam.com.ar/advf/documentos/2013/02/512e871662d27.pdf

Buergentha, T. (2017). Comparando Transparencia. DPFL.

Buscaglia, E. (1997). Los Principales Obstáculos de la Reforma Judicial en América Latina. Banco Interamericano de Desarrollo .

Castells, M. (1998). Globalización, sociedad y política en la era de la Información. Revista Bitácora, 4253.

Castillo, M. (2013). Ley vs. realidad: Independencia y transparencia de la justicia en Centroamérica y Panamá. Due Process of Law Foundation.

Castro, F., \& García, G. (2006). Indicadores, Transparencia y Accountability para la Reforma Procesal Civil. Pontificia Universidad Católica de Chile.

CEPAL. (1998). La Inversión Extranjera en América Latina y el Caribe 1997.Comisión Económica para América Latina y el Caribe.

Cipoletta, G. (2009). La seguridad social en la República Argentina. Revista Latinoamericana de Derecho Social, (8), 3-51.

Consentino, R. (2009). Técnicas de Gestión Judicial y Eficacia del Proceso. Dirección Nacional del Sistema Argentino

Contini, F., \& Velicogna, M. (2012). Del acceso a la información al acceso a la justicia: Diez años de ejustice en Europa. MARCO, 30-47.

Creswell, J. W. (1994). Diseño de investigación: Aproximaciones cualitativas y cuantitativas. Thousand Oaks: Sage.

Delgado, J., \& Díaz, I. (2011). La Unificación de Jurisprudencia Pretendida por el Recurso Extraordinario. Ventajas y Problemas. Revista de Derecho, (2), 275-304.

Dory, R. (2009). Comprendiendo las tecnologías de la información para la resolución de conflictos. Sistemas Judiciales, 18-29.

Duce, M. (2004). La Reforma Procesal Penal Chilena: gestación y avance de un proceso de transformación en marcha. En En Busca de Una Justicia Distinta: Experiencias de Reforma en América Latina (pp.195-248). Consorcio Justicia Viva.

Estrada, U., \& Gleidy, A. (2015). La defensa jurídica y patrimonial del Estado colombiano: un reto institucional en el marco de la gubernamentalidad. Ánfora, 61-83.

Franco, S. (2017). Transparencia y ética judicia. Cuestiones Constitucionales, (36), 143-166.

Garavano, G., \& Palma, L. (2002). La capacitación judicial en la Argentina. Ideas para su potenciación. Realidad Judicial, (4).

García, P. (2012). Herramientas de gestión judicial para los juzgados de familia. Revista de Derecho, (25), 155-181.

Gregorio, C. (2006). Gestión judicial y reforma de la administración de justicia en América Latina. Banco Interamericano de Desarrollo.

Hernández Sampieri, R., Fernández Collado, C., \& Baptista Lucio, M. (2014). Metodología de la investigación. McGrawHill.

Hernandez, R. (2017). La evaluación del desempeño del talento humano en el poder judicial. Universidad de Costa Rica.

Herrera Romero, L. (2015). La Calidad en el Sistema de Administración de Justicia. Universidad Esan.

Jiménez, M., A., M., \& A., G. (2006). Juzgados y documentación clínica. An. Sist. Sanit, 29 (2), 253 262.

Jiménez, R. (2005). Administración de Justicia y nuevas tecnologías. Anuario multidisciplinar para la modernización de las Administraciones Públicas, (1), 63-80.

Ledesma, Á. (2017). Manual para formadores en gestión judicial estratégica. SAIJ de la Dirección Nacional del Sistema Argentino. 
Treviño., R., R., Ortiz., T., G., \& Maldonado., S., H.

Linares, S. (2008). La administración de las políticas de justicia en América Latina: nuevos horizontes de reforma. Revista Iberoamericana, 8 (31), 172-175.

Lisa, H. (2014). Jueces y política en democracia y dictadura: Lecciones desde Chile. FLACSO.

Londoño, R. (2010). El uso de las TIC en el proceso judicial: una propuesta de justicia en línea. Revista de la facultad de derecho y ciencias políticas, 40 (112), 123-142.

López, G. (2005). La profesionalización del Poder Judicial. Revista buen gobierno 127-141.

Manjónm, A. (2008). ¿Son vinculantes los acuerdos del pleno no jurisdiccional de la sala segunda del ts?. Revista Electrónica de Ciencia Penal y Criminología, 10, (2), 1-25.

Mejía, B. (2001). Corrupción Judicial en Perú: Causas, Formas y Alternativas. Derecho \& sociedad, (17), 208-215.

Mora, J. (2018). Las juntas de letrados de la administración de justicia: análisis de su régimen jurídico y apuntes para su reforma. especial referencia a su valor como instrumento para la unificación de criterios. Acta Judicial, (2), 41-68.

Mora, L. (2005). Algunas observaciones sobre gestión judicial en América Latina. América Latina Hoy, (39), 125-140.

Nieto, S. (2007). La reforma del Poder Judicial en el marco de la reforma del Estado: temas para discusión. El Cotidiano, 22 (146), 73-79.

Opazo, F. (2014). Gestión en los tribunales ordinarios. Universidad de Chile.

Orme, B. (2017). Mejores prácticas para orientar el diálogo entre el Poder Judicial y la prensa. Organización de las Naciones Unidas para la Educación, la Ciencia y la Cultura.

Palma, L. (2007). La capacitación judicial y sus nuevos caminos. Diario.

Palma, L. (2017). Modernización judicial, gestión y administración en América Latina. Sciencie Direct, (72), 149-203.

Peña, J. (2010). La gestión judicial y su implementacion dentro del poder judicial de la federacion. Revista Juridica, (29), 265-277.

Peñaranda, H. (2010). Principios procesales del amparo constitucional. Nómadas, 26 (2), 1-78.

Pineda, B. (2007). Las Tecnologías en la aplicación de la Justicia y los Derechos Humanos. Revista Iberoamericana de Producción Académica y Gestión Educativa, (2), 1-35.

Raynaud, A. (2009). Necesidad de una profunda reforma procesal laboral en América Latina. Congreso Nicaragüense del Derecho del Trabajo y la Seguridad Social.

Riego, C. (2003). Seguimiento de los Procesos de Reforma Judicial en América Latina. Sistemas Judiciales, 34-73.

Rodriguez, M. (2013). La utilización de los avances tecnológicos para la solución de conflictos: Algunas novedades respecto a la digitalización del procedimiento judicial y la implementación del expediente electrónico. Revista Electrónica del Instituto de Investigaciones, (11), 147-182.

Ruiz, A. (2010). Principios procesales necesarios en la administración de justicia en asuntos laborales y por prestaciones de la seguridad social en méxico. Revista Latinoamericana de Derecho Social, (10), 203-238.

Salazar, M. (2014). Autonomía e independencia del poder judicial Peruano en un estado social y democrático de derecho. Ciencia y Tecnología, 10 (2), 147-161.

Toledo, I. (2014). El marco institucional contra la corrupción. Mastergraf.

Torres, J. (2014). Avances y modernización en la gestión judicial. Corte Suprema de Justicia de la República del Paraguay.

Ubertone, F. (2006). Carrera de Especialización en Administración de Justicia. Revista sobre enseñanza del Derecho, 9 (17), 431-442.

Vallejo, C. (2013). La transparencia y la rendición de cuentas como herramientas de cambio de la gestión judicial en el consejode la judicatura federal. Senado de la Republica.

204 Revista Política, Globalidad y Ciudadanía | Vol. 7, Núm. 14, junio - diciembre 2021 | ISSN 2395-8448 | http://revpoliticas.uanl.mx/ 
Vargas, J., Peña, C., \& Correa, J. (2001). El rol del Estado y el mercado en la justicia. Cuadernos de análisis jurídico.

Vargas, L. (2003). Eficiencia en la justicia. Instituto de Investigaciones Jurídicas.

Vergara, A. (2015). Los jueces en la era del derecho democrático. Especialización, principios y activismo judicial. Centro de Políticas Públicas UC, (83), 1-12.

Vivianco, A. (2008). Transparencia de la función pública y accesoa la información de la administración del estado: una normativa para chile. Revista Chilena de Derecho, 35 (2), 371 - 398.

William Collins Sons \& Co. (2005). Collins Spanish Dictionary. Harper Collins Publishers. 